\title{
SYSTEMATIC SEARCH FOR QUASAR CANDIDATES USING THE MAMA MICRODENSITOMETER
}

\author{
O. MOREAU ${ }^{1 \dagger}$, E. GOSSET ${ }^{1}$, H. REBOUL ${ }^{2}$ and C. VANDERRIEST ${ }^{3}$ \\ ${ }^{1}$ Institut d'Astrophysique, Université de Liège, Avenue de Cointe 5, B-4000 Liege, \\ Belgium \\ ${ }^{2}$ Laboratoire d'Astronomie, Université Montpellier II, F-34095 Montpellier Cedex \\ 5, France \\ ${ }^{3}$ Observatoire de Paris-Meudon (DAEC), 5 Place Janssen, F-92195 Meudon Cedex, \\ France
}

\begin{abstract}
Increasing the density of known quasars in wide fields is of great interest, especially in the frame of the search for a possible very-large-scale structuring of the Universe. We report here on the status of two photographic systematic surveys for quasar candidates that we are conducting.
\end{abstract}

\section{Scientific Interest}

In order to obtain a better understanding of the physics, the evolution and the spacetime distribution of quasars, it is still necessary to increase the number of known objects, especially within wide regions and on the basis of homogeneous and objective criteria. One of the main interests is the possibility, provided large, deep and homogeneous quasar surveys become available, to study the distribution of matter in the Universe at intermediate redshifts; this is especially important in order to make a link between the high degree of structuring locally displayed by the galaxy distribution and the homogeneous universe of the most widely used cosmological models. Moreover, there is nowadays an increasing interest for high redshift quasars which justifies systematic searches sensitive to this redshift range. Last but not least, quasar surveys are also a direct source of new potential gravitational mirage candidates (see e.g. Moreau et al. 1993) and of new cases of binary quasars in which cosmological interest is also high, as they possibly allow the determination of $q_{0}$ (Reboul et al. 1986).

\section{Method}

Schmidt photographic plates (see sections 3 and 4) are fully digitised with the MAMA high-speed and high-accuracy measuring machine of Centre d'Analyse de Images located at Observatoire de Paris (see Guibert \& Moreau 1991; Moreau 1992) with a pixel size of $10 \mu \mathrm{m}$ corresponding to 0.67 arcsec on the plates. A basic thresholding algorithm is applied to the digitized images,

${ }^{\dagger}$ European Space Agency fellow

740

H. T. MacGillivray et al. (eds.), Astronomy from Wide-Field Imaging, 740-742.

(C) 1994 IAU. Printed in the Netherlands. 
leading to an exhaustive catalogue of objects detected on the plates. The catalogues thus produced are then calibrated using standard stars (Berger et al. 1991; Moreau 1992) in order to give a celestial position (accurate to about $0.3 \mathrm{arcsec}$ ) and a magnitude (with an accuracy of about $0.1 \mathrm{mag}$ ) for each object. Cross-identifications of objects between catalogues from different plates of the same field lead to a multi-colour catalogue if plates taken in different passbands at the same epoch are available and/or to a catalogue of photometric variabilities if one benefits from plates taken through the same filter at different epochs.

Quasar candidates are systematically selected among the exhaustive catalogues produced, with colour and/or variability criteria. With U, B and V plates, a colour survey is sensible to rather low redshift quasars $(\mathrm{z} \leq 2.2)$ while a UBVR - or better UBVRI - one will yield high redshift candidates (about $\mathrm{z} \geq 3$ ). The variability criterion is far less sensitive to redshift biases provided that the duration of the survey is long enough to cope with the $(1+z)$ cosmological time dilation effect. It is therefore an interesting complementary way of selecting quasars; however, in order to correctly separate them from variable stars, one needs a large number of plates, with an adequate time sampling. All the selected candidates must of course be confirmed by spectroscopy.

Two deep and homogeneous surveys for quasars are being conducted in collaboration with Centre d'Analyse des Images; one (Liège-Meudon-Montpellier) covers a wide 300 sq. degree region around the North Galactic Pole (NGP) while the other one (Liege) deals with three 30 sq. degree fields (labelled NGC 450, NGC 520 and ESO 300) observable from the southern hemisphere.

\section{A Survey in 300 Square Degrees at the NGP}

Considerable high-quality photographic plate material has been taken by J. Berger with the Palomar Schmidt telescope in 1962. Eight 40 sq. degree PSS contiguous fields have been covered in the $U, B, V$ and, for some, $R$ passbands, allowing the systematic multi-colour survey of a wide $\simeq 300 \mathrm{sq}$. degree region ranging from $\alpha_{2000}=12 \mathrm{~h} 01 \mathrm{~min}$ to $13 \mathrm{~h} 47 \mathrm{~min}$ and from $\delta_{2000}=+20^{\circ} 03^{\prime}$ to $+32^{\circ} 25^{\prime}$. Limiting magnitudes of the plates are of the order of 20 in each passband. In addition, we have obtained yearly since 1990 (and are still regularly requesting) 27 sq. degree V plates from the Schmidt telescope of Observatoire de la Côte d'Azur (OCA, France) in order to get more and more epochs for variability studies. Five plates of field PSS $13^{h} 00+30^{\circ}$ (containing SA57) have already been scanned and, at this time, three of them (Palomar U, Palomar V and OCA V) have been reduced. About 2000 quasar candidates have been extracted with colour and/or variability criteria (Moreau 1992). Observing time for follow-up spectroscopy has been requested at the Canada-France-Hawaii telescope with use of the MOS multi-object spectrograph, this step being indispensable to tackle the scientific questions quoted in section 1 . It is worthy of note that part of the candidates are being correlated with the ROSAT catalogue of X-ray sources. Moreover, in field PSS $13^{h} 26+30^{\circ}$ (containing M 3), all the Palomar plates have been digitized but not yet reduced. 


\section{The Liège Quasar Survey}

A UBVRI + variability systematic survey has been undertaken in three fields: NGC 520, NGC 450 and ESO 300 in which visual searches for quasar candidates have already been performed, mainly on the basis of dual exposure (U/B) plates (Swings et al. 1988). As a matter of fact, following this first visual step, spectra have been taken, mainly with the Irenee Dupont $2.55 \mathrm{~m}$ telescope (Las Campanas, Chile), leading to the confirmation of more than 100 quasars and we are now preparing MAMA photometry and astrometry of all the candidates visually selected in fields NGC 450 and NGC 520. A large number of ESO Schmidt plates have been obtained (and are still requested) for that systematic survey project: 10 plates (UBVR) in field NGC 520 and 10 (UBVRI) in both NGC 450 and ESO 300 fields; limiting magnitudes are somewhat deeper than for the plates in NGP fields. Note that R and I plates are expected to be especially useful for the selection of high-redshift quasar candidates. Four ESO plates (UBVR) of field NGC 450 have already been scanned and presently only the $\mathrm{B}$ one has been reduced.

\section{Acknowledgements}

We would like to thank J. Berger who kindly made available to us the photographic plates he took at Palomar. Our gratitude is also due to the MAMA scientific and technical team for the constant and efficient assistance we are receiving. Thanks are also expressed to the ESO and OCA Schmidt teams for the good plates they are providing. This research is supported in part by contracts ARC 90/94-140 'Action de Recherche Concertee de la Communauté Française' and SC-005 'Service Centers and Research Networks of the Science Policy Programming Services of the Prime Minister's Office’ (Belgium).

\section{References}

Berger, J., Cordoni, J.-P., Fringant, A.-M., Guibert, J., Moreau, O., Reboul, H. and Vanderriest, C., 1991. Astron. Astrophys. Suppl. Ser., 87, 389.

Guibert, J. and Moreau, O., 1991. The Messenger, 64, 69.

Moreau, O., 1992. Thèse de Doctorat, Université Paris 7 (France)

Moreau, O., Claeskens, J.-F., Gosset, E., Reboul, H. and Surdej, J., 1993. In 'Gravitational Lensing in the Universe', 31st Liège Int. Astroph. Coll., in press.

Reboul, H., Fringant, A.-M. and Vanderriest, C., 1986. In 'Quasars', IAU Symposium No 119, p. 547.

Swings, J.-P., Surdej, J. and Gosset, E., 1988. ASP Conf. Series, 2, 61. 


\title{
ASTRONOMY FROM WIDE-FIELD
}

\section{IMAGING}

\author{
Part Sixteen:
}

\section{CONFERENCE SUMMARY AND RESOLUTIONS}

